\title{
Deletion polymorphism at chromosome 3q26.1 and oral squamous cell carcinoma
}

\author{
HOMARE KAWACHI $^{1}$, KEISUKE SUGAHARA ${ }^{1}$, YASUTAKA NAKAMURA ${ }^{2}$, AKIRA KATAKURA ${ }^{1,3}$, \\ KIYOSHI MINAGUCHI $^{2}$ and TAKAHIKO SHIBAHARA ${ }^{1}$ \\ Departments of ${ }^{1}$ Oral and Maxillofacial Surgery, ${ }^{2}$ Forensic Odontology, \\ and ${ }^{3}$ Oral Medicine, Tokyo Dental College, Tokyo, Japan
}

Received September 12, 2012; Accepted November 2, 2012

DOI: $10.3892 /$ ijo.2012.1749

\begin{abstract}
Several recent studies have investigated DNA instability in malignancies including deletions and duplications of part of the chromosome using array-based comparative genomic hybridization $(\mathrm{CGH})$ analysis. Using the same approach on oral squamous cell carcinoma (OSCC) tissue samples, we found a frequent deletion at chromosome $3 \mathrm{q} 26.1$ in OSCC patients; this polymorphism showed a gene frequency of 0.293-0.368 in healthy volunteers $(n=60)$ and $0.129-0.195$ in OSCC patients $(n=54)$. Detailed analysis around the polymorphic region revealed the deletion breakage point. A significant association of gene frequency for the deletion polymorphism between healthy volunteers and patients implicated genetic factors related to this polymorphism in the development of OSCC. Currently, no gene is predicted to lie within the 3,606-kbp region around the polymorphism. Thus, although a single-gene model could not explain the occurrence of OSCC, we believe that examining this polymorphism could be useful in identifying risk factors for OSCC.
\end{abstract}

\section{Introduction}

Recent years have seen an increased incidence of oral squamous cell carcinoma (OSCC) cases in Japan, coinciding with population aging. OSCC accounts for 1-2\% of all cancers, and approximately $40 \%$ of head and neck cancers. The ratio between men and women for OSCC is $3: 2$. Globally, the prevalence of OSCC is high in nations with high levels of alcohol and tobacco use (1-3). Pathologically, $80 \%$ of oral cancers are squamous cell carcinoma $(4,5)$.

Like many other cancers, the occurrence of OSCC is thought to be intricately associated with both genetic and environmental factors. As the entry of the gastrointestinal system,

Correspondence to: Professor Takahiko Shibahara, Department of Oral and Maxillofacial Surgery, Tokyo Dental College, 1-2-2 Masago, Mihama-ku, Chiba 261-8502, Japan

E-mail: sibahara@tdc.ac.jp

Key words: oral squamous cell carcinoma, 3q26.1, deletion, polymorphism, real-time PCR the oral cavity is exposed to various environmental insults such as chemical stimuli from alcohol and tobacco $(6,7)$, food, and physical stimuli from dental caries and faulty dental prostheses. All such insults are considered carcinogenic risk factors for the oral mucosal membranes (8-11).

A review of chromosomal aberrations in oral or head and neck squamous cell carcinoma concluded that the most significant findings are chromosomal changes, suggesting the involvement of tumor suppressor genes (TSGs) (12). In studying the relationship between OSCC metastasis and chromosomal aberration, we found frequent deletion of a probe on chromosome 3 by array-based comparative genomic hybridization (CGH) analysis of OSCC tissues. The deletion was not related to metastasis (13). The present study further examined how the identified chromosomal deletion relates to OSCC.

\section{Materials and methods}

Samples. Twenty tumor tissue samples were used for extraction of DNA for array-based CGH analysis and also for real-time PCR. DNA for real-time PCR was also collected from another 11 tumor tissues, 31 marginal tissues around the tumors, and 31 peripheral blood samples from healthy volunteers. A total of 54 and 60 peripheral blood samples were further obtained from different OSCC patients and healthy volunteers, respectively. All OSCC patients underwent surgical resection at Tokyo Dental College, Chiba, Japan, between April 2007 and July 2010. Healthy volunteers were selected from workers at the same college. Written informed consent was obtained from all participants in accordance with the Ethical Guidelines on the Use of Human Tissues. The Ethics Committee of Tokyo Dental College approved the study (approval no. 205). The 54 OSCC patients were aged from 43 to 89 years, and the 60 healthy volunteers were aged from 24 to 48 years. Table I summarizes the clinical characteristics of the study participants.

DNA extraction. Sample for DNA extraction were stored at $-20^{\circ} \mathrm{C}$ until use. Genomic DNA was extracted from peripheral blood using a QIAamp DNA blood midi kit (Qiagen, Valencia, CA) and from tissues using a QIAamp DNA Maxi kit (Qiagen) according to the instructions supplied by the manufacturer. The DNA was quantified using a Nano drop ${ }^{\circledR}$ (ND-1000 Spectrophotometer, Thermo Fisher Scientific, Waltham, MA). 
Table I. Clinical characteristics of OSCC patients and healthy volunteers.

\begin{tabular}{|c|c|}
\hline & OSCC patients $(n=54)$ \\
\hline Age (years; means \pm SD) & $66 \pm 11.67$ \\
\hline \multicolumn{2}{|l|}{ Gender } \\
\hline Male & 27 \\
\hline Female & 27 \\
\hline \multicolumn{2}{|l|}{ Tobacco consumption } \\
\hline Negative & 22 \\
\hline Positive & 32 \\
\hline \multicolumn{2}{|l|}{ Alcohol consumption } \\
\hline Negative & 32 \\
\hline Positive & 22 \\
\hline \multicolumn{2}{|l|}{ Site } \\
\hline Tongue & 28 \\
\hline Gingiva & 16 \\
\hline Palate & 1 \\
\hline Buccal mucosa & 5 \\
\hline Oral floor & 4 \\
\hline \multicolumn{2}{|l|}{$\mathrm{T}$ classification } \\
\hline $\mathrm{T} 1$ & 18 \\
\hline $\mathrm{T} 2$ & 23 \\
\hline $\mathrm{T} 3$ & 5 \\
\hline $\mathrm{T} 4$ & 8 \\
\hline \multicolumn{2}{|l|}{ Stage classification } \\
\hline I & 16 \\
\hline II & 16 \\
\hline III & 9 \\
\hline IV & 13 \\
\hline
\end{tabular}

Healthy volunteers $(n=60)$

\begin{tabular}{lc}
\hline Age (years; means \pm SD) & $29 \pm 4.57$ \\
Gender & 46 \\
Male & 14 \\
Female & \\
Tobacco consumption & 41 \\
$\quad$ Negative & 19 \\
Positive & \\
Alcohol consumption & 1 \\
Negative & 59 \\
Positive & \\
\hline
\end{tabular}

Array-based CGH analysis. Array-based CGH analysis was performed on primary tumor tissues using a Human Genome CGH Microarray Kit 44K (Agilent Technologies, Santa Clara, CA), containing in situ-synthesized 60-mer oligonucleotides representing 42,494 unique probes for human genes. Labeling and hybridization were performed essentially as described previously with the following modifications. We amplified $100 \mathrm{ng}$ each of reference (male or female human genomic DNA;
Table II. Gene polymorphism at chromosome 3q26.1 between healthy volunteers and OSCC patients by real-time PCR.

\begin{tabular}{lccc}
\hline & Healthy volunteers & OSCC patients & P-value \\
\hline Normal & 27 & 10 & $<0.05$ \\
Loss & 4 & 21 & \\
\hline
\end{tabular}

Promega, Madison, WI) and tumor DNA with Phi29 DNA polymerase according to the protocols provided by the supplier (Qiagen). DNA was then digested with AluI (50 units) and RsaI (50 units; Promega) for $2 \mathrm{~h}$ at $37^{\circ} \mathrm{C}$. Digests were filtered using the QIAprep Spin Miniprep kit (Qiagen) and then verified on a Bioanalyzer (Agilent Technologies). Fluorescent labeling reactions to make hybridization probes were performed with $7 \mu \mathrm{g}$ of purified restricted DNA using a BioPrime array-based CGH genomic labeling kit (Invitrogen), according to the instructions provided by the manufacturer, in a volume of $50 \mu \mathrm{l}$ with a modified dUTP pool containing $120 \mu \mathrm{M}$ each of dATP, dGTP, and dCTP; $60 \mu \mathrm{M}$ dTTP; and $60 \mu \mathrm{M}$ Cy5-dUTP or Cy3-dUTP (Perkin-Elmer, Waltham, MA). Labeled reference and tumor DNA probes were subsequently mixed and filtered through a Microcon YM-30 column (Millipore, Billerica, MA) then verified on a Bioanalyzer (Agilent Technologies). To the mixtures were added $50 \mu \mathrm{g}$ of Human Cot-1 DNA (Invitrogen)/Agilent 10X Blocking Agent/Agilent 2 Hybridization Buffer. Before hybridization to the array, the hybridization mixtures were denatured at $95^{\circ} \mathrm{C}$ for $3 \mathrm{~min}$, and then incubated at $37^{\circ} \mathrm{C}$ for $30 \mathrm{~min}$. The mixtures were centrifuged at $17,900 \mathrm{x} \mathrm{g}$ for $1 \mathrm{~min}$ to remove any precipitate, and then applied to the array using an Agilent microarray hybridization chamber. Hybridization was carried out for $40 \mathrm{~h}$ at $65^{\circ} \mathrm{C}$ in a rotating oven (Robbins Scientific, Mountain View, CA) at $20 \mathrm{rpm}$. The arrays were then disassembled in $0.5 \mathrm{X} \mathrm{SSC} / 0.005 \%$ Triton X-102 at room temperature, washed for $5 \mathrm{~min}$ at room temperature in wash 1 , and then incubated for $1 \mathrm{~min}$ at $37^{\circ} \mathrm{C}$ in $0.1 \mathrm{X} \mathrm{SSC} / 0.005 \%$ Triton $\mathrm{X}-102$ (wash 2). Slides were dried and scanned using an Agilent G2565B DNA microarray scanner $(14,15)$.

Real-time PCR. Real-time PCR was performed using tumor, marginal tissue, and blood samples with SYBR Green I fluorescence detection on a Light Cycler 480 (Roche Diagnostics, Basel, Switzerland). Oligonucleotide primers for real-time PCR were designed using Primer software (Whitehead Institute for Biomedical Research), and uniqueness in the human genome was checked by a BLAST search. Nucleotide sequences of oligonucleotide primers were: F, 5'-CGGGGAGTTTGATTTTCACT-3' and R, 5'-CGCTGTATGGTTGTCTTGTTG-3'. The 20- $\mu 1$ reaction mixture consisted of $10 \mu 1$ XX IQ SYBR Green Supermix (Bio-Rad Laboratories, Hercules, CA), $2.5 \mathrm{ng}$ genomic DNA, and $800 \mathrm{nM}$ of each PCR primer. The reaction mixtures were heated at $95^{\circ} \mathrm{C}$ for $10 \mathrm{~min}$ and then subjected to 40 rounds of two-step temperature cycling $\left(95^{\circ} \mathrm{C}\right.$ for $15 \mathrm{sec}$ and $65^{\circ} \mathrm{C}$ for $60 \mathrm{sec}$ ) (16). The crossing point for each amplification curve was determined by the second derivative maximum method. The standard curve method using separate reaction wells was applied for relative quantification. We used NAGK (N-acetyl glucosamine kinase, NAGK) as internal reference loci (17). 
Detection method for comparison and typing of polymorphic regions. Table II details the PCR primer sequences used for amplifying the regions around the hybridized probe sequence by array-based CGH and the PCR target sequences. PCR amplification was performed in a $30-\mu 1$ mixture containing $10 \mathrm{ng}$ genomic DNA, $10 \mathrm{mM}$ Tris- $\mathrm{HCl}$ at $\mathrm{pH} 8.3,50 \mathrm{mM} \mathrm{KCl}, 2.5 \mathrm{mM}$ $\mathrm{MgCl}_{2}, 0.02 \%$ gelatin, $200 \mu \mathrm{M}$ dNTP, $400 \mathrm{nM}$ of each primer, and 1.25 U AmpliTaq Gold (Applied Biosystems). A two-step PCR amplification process was used: $95^{\circ} \mathrm{C}$ for $11 \mathrm{~min}$, followed by 28 cycles of denaturation at $95^{\circ} \mathrm{C}$ for $40 \mathrm{sec}$, and annealing and extension at $59^{\circ} \mathrm{C}$ for $105 \mathrm{sec}$. After the 28th cycles, a final extension step was performed at an annealing/extension temperature at $60^{\circ} \mathrm{C}$ for $10 \mathrm{~min}$. PCR products were separated on $8 \%$ polyacrylamide gels, and all products were visualized by silver staining (18).

Sequence analysis. PCR for sequencing was performed using the BigDye $^{\mathrm{TM}}$ Terminator v1.1 Cycle Sequencing Ready Reaction Kit (Applied Biosystems), and the products were purified using a PCR purification kit (Invitrogen). Excess dye was removed using Performa DTR gel filtration cartridges (EdgeBio, www. edgebio.com). Sequence analysis was performed on an ABI 3100 DNA sequencer.

Statistical analysis. The $\chi^{2}$ test was used for comparisons between the presence and absence of deleted regions, using a $2 \times 2$ contingency table. The homogeneity test for gene frequencies was performed according to Samaneh et al (2010) (19).

\section{Results}

Array-based CGH analysis. The array-based CGH analysis was performed on 20 OSCC tumor tissue samples using Feature Extraction software (version 8.5.1.1, Agilent Technologies) and the linear normalization method for background subtraction. Datasets created from the dye-swap experiments were averaged and then further analyzed using CGH Analytics software (version 3.4, Agilent Technologies). Although we were originally investigating the relationship between metastasis and chromosomal aberration, a frequent deletion on chromosome 3 showing up on the array-based CGH analysis was not associated with any clinical features of metastasis (13). This deletion was found in 70\% (14/20) of DNA extracts from OSCC tissues samples on the long arm of chromosome 3 (Fig. 1). Detailed analysis mapped the region to chromosome 3 q26.1. Because the deletion frequency was so high in OSCC tissues, we next studied its relationship to other clinical features.

Examination of the deleted region by real-time PCR. We next applied real-time PCR to further study the deleted region at chromosome 3q26.1 identified by array-based CGH (arraybased CGH probe sequence $=3$ q26.1-pro-seq). Real-time PCR primers were initially constructed to amplify the probe region, 5'-flanking region, and 3'-flanking region of the probe. However, because the most similar results to those on arraybased $\mathrm{CGH}$ analysis were obtained using primers for the 5'-flanking region, we used these primers for further studies. DNA samples were isolated from another 11 OSCC tissues and from marginal tissues around the tumor in the 31 OSCC patients. When real-time PCR was conducted for DNA isolated from tumor and marginal tissues, the same tendency for quantification was obtained from both sample types. Assuming that marginal tissues contained mainly normal tissue, the real-time PCR suggested that the deletion of 3q26.1-pro-seq is not due to chromosomal aberration resulting from carcinogenesis, but instead occurs through inherent genetic variation.

To explore if the deletion around 3q26.1-pro-seq is genetically restricted to OSCC patients, we collected DNA samples from 31 healthy volunteers as controls and performed real-time PCR for the 5'-flanking region of 3q26.1-pro-seq (Fig. 2). If the deletion was indeed genetic variation, the real-time PCR results must be classified into three types: complete deletion, hemizygote, and non-deletion homozygote. Although the distribution of $\mathrm{Cp}$ values could not be clearly divided, we classified them into three groups using tentative threshold values. The average Cp values for these groups were $26.5 \pm 2,30.5 \pm 2$, and $34.5 \pm 2$, respectively. The first type corresponded to homozygotes for 3q26.1-pro-seq (+), the second type to heterozygotes for $3 q 26.1$-pro-seq, and the third type to 3q26.1-pro-seq (-) homozygotes. Because the boundary lines of these values were not clear-cut, we classified them into 3q26.1-pro-seq-positive type (+) (non-deletion homozygote and heterozygote) and 3q26.1pro-seq-loss type (-), to compare frequencies between healthy and OSCC patient groups by the $\chi^{2}$ test (Table II). A statistically significant difference was observed regarding presence and loss of 3q26.1-pro-seq between the OSCC patients and healthy volunteers $\left(\mathrm{P}=8.1 \times \mathrm{E}^{-06}\right.$; Table II). Loss of 3q26.1-pro-seq was more common in OSCC patients.

Detection and evaluation of genetic variation. Although significant association was observed in the distribution of 3q26.1-pro-seq-positive and -negative individuals, the total number of samples was small (31 per group). In addition, the real-time PCR typing results were ambiguous because the boundaries between genotypes were unclear. We therefore sought to establish reliable typing methods and increase the number of samples to confirm if the suspected association was conserved in new population samples.

We first compared the presence and absence of the 3q26.1-pro-seq sequence using simple PCR amplification followed by native polyacrylamide gel electrophoresis (Fig. 3). First, we constructed primers (Fig. 3, primer 3-4) to amplify the fragment (141-bp fragment) that included the target region for real-time PCR (71-bp fragment amplifiable by primer 1 and 2) (Fig. 3). When a 141-bp fragment was amplified by PCR in 35 cycles of amplification including $2.5 \mathrm{U}$ of AmpliTaq Gold, strong positive bands or faint bands corresponding to the same migration position as the target PCR product appeared in many samples, and negative samples were rare. Sequencing of the faint band and the intensified band determined that the amplified fragments were identical. National Center for Biotechnology Information (NCBI) blast searching using primers 3 and 4 revealed no similar sequences to the 141-bp fragment on chromosome 3 . This suggested that the faint band was not amplified from different similar sequences in the genome, and contamination with foreign DNA occurred in the course of DNA collection and experiments. All reagents used for the PCR amplification were subsequently replaced to identify the possible source of contamination. Indeed, we employed a different company to synthesize the oligonucleotide primers 


\section{Chromosome 3}
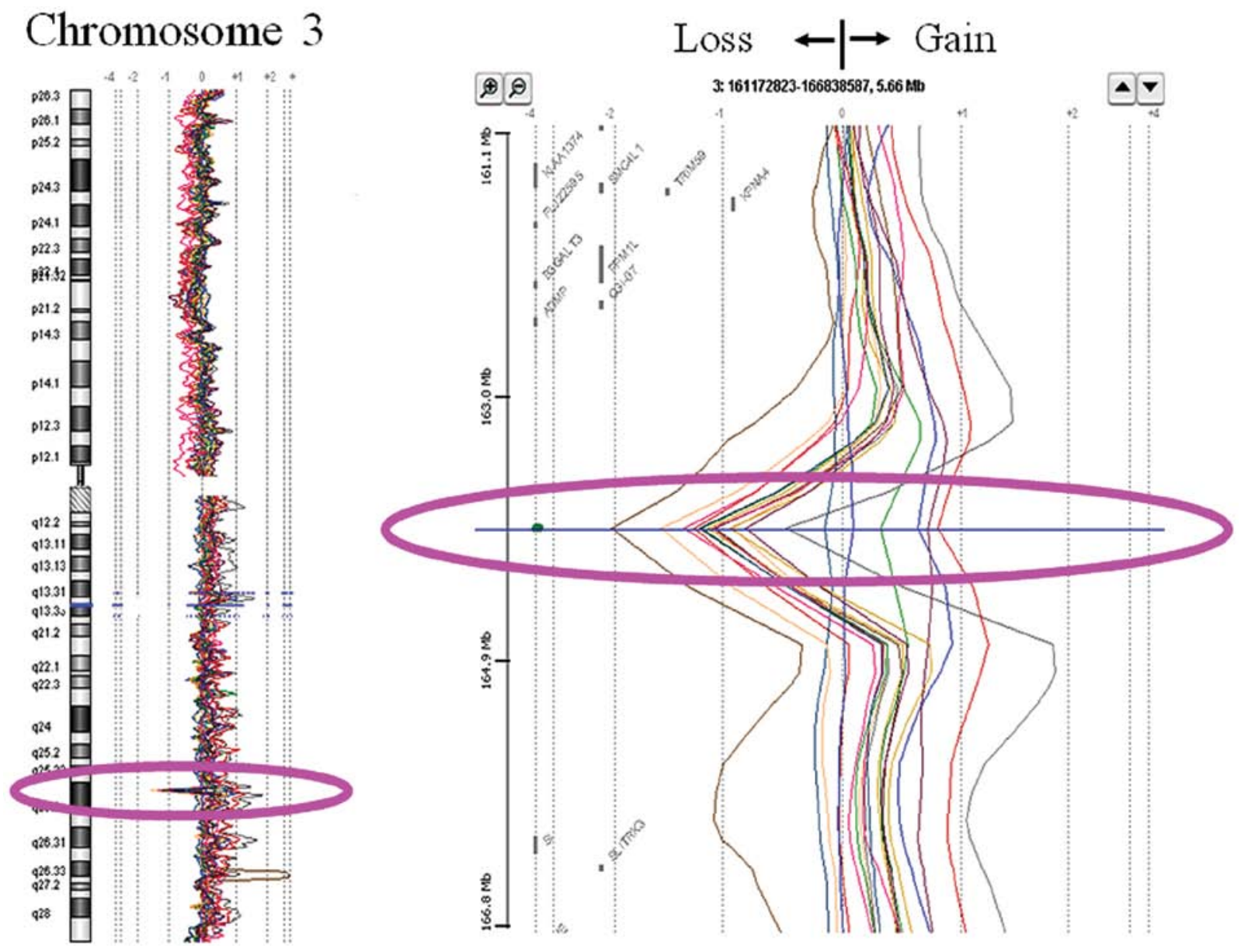

Figure 1. Schematic illustration of cytogenetic alteration in chromosome $3 q$ in OSCC. DNA obtained from OSCC tissues samples. The portion of chromosome 3 q26.1 was deficient in 14 of 20 people (70\%).
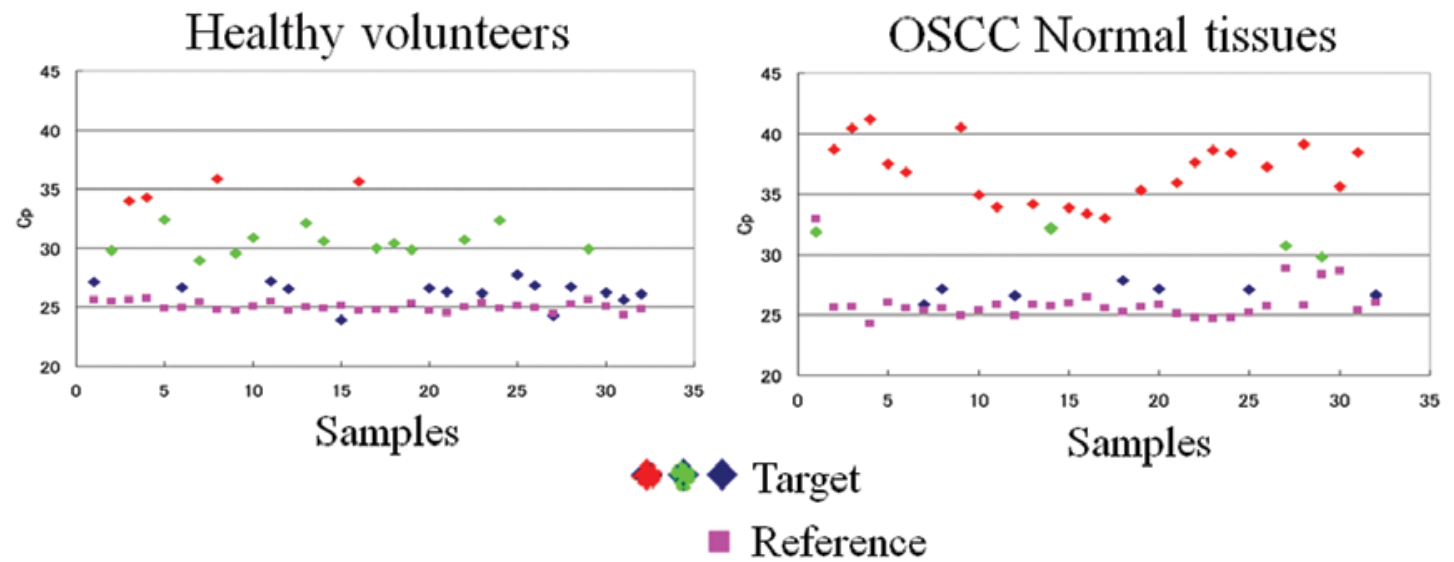

Figure 2. Schematic representation of the DNA copy number differences between blood sample from a healthy volunteer and normal tissues of a patient with OSCC, detected at 3q26.1 by real-time PCR. Pink, reference primer. Red, green, and blue bands, target primers.

used to amplify the 141-bp products. However, similar results were obtained, suggesting that the contamination did not occur during PCR amplification.

Next, we constructed primers to amplify a large fragment (294-bp) including the 141-bp fragment and 3q26.1-pro-seq to ascertain whether the unstable amplification among samples was due to sequence heterogeneity around the 141-bp fragment (Fig. 3). PCR amplification was then performed using three different sets of primers that each amplified the 141-bp fragment, the real-time PCR fragment (designated as a 71-bp fragment amplified by primer 1-2 in the following explanation), and the 294-bp fragment (Fig. 3 and Table III). The amplified 


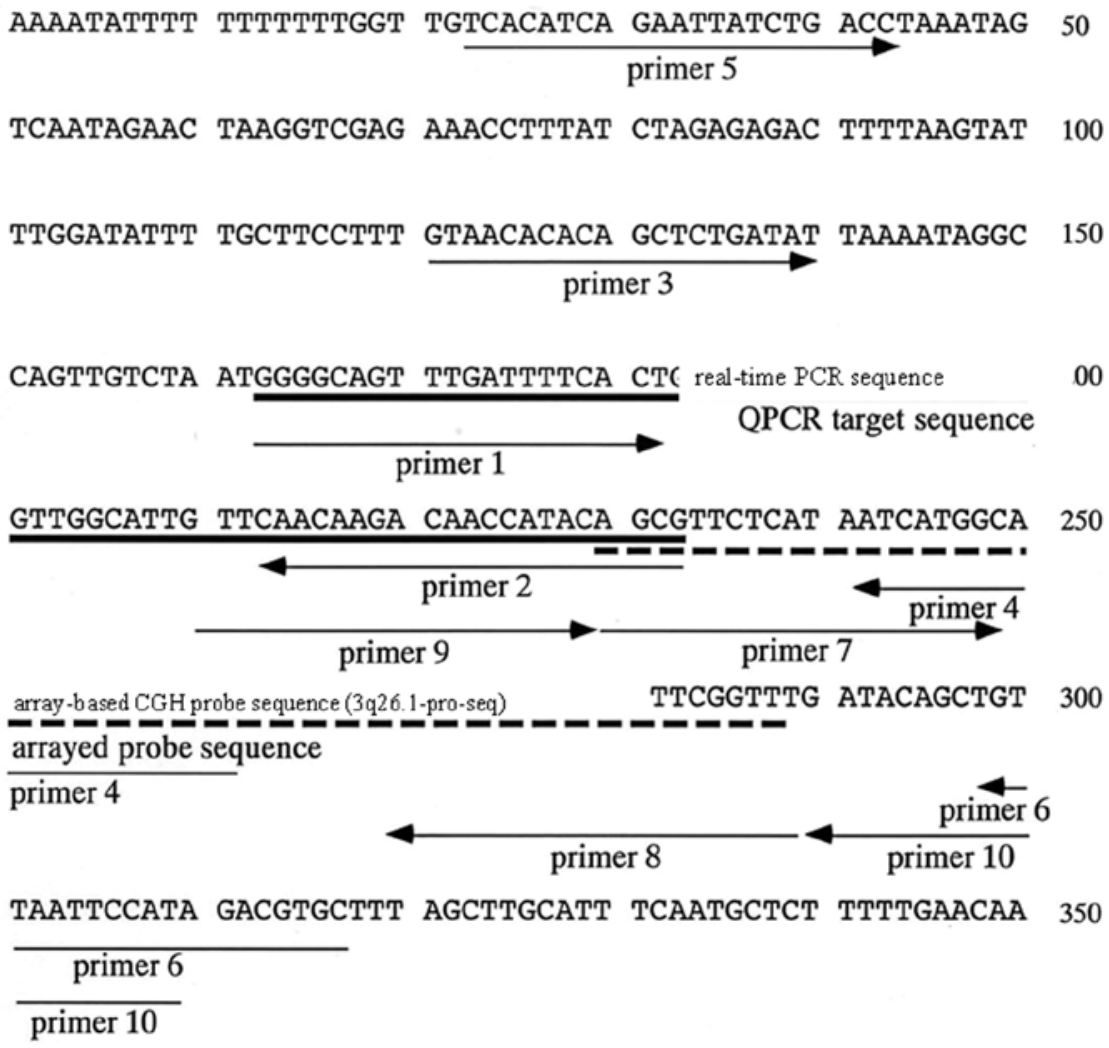

Figure 3. Nucleotide sequences around the real-time PCR target region and array-based CGH probe sequence. Primers (1-10) used to amplify various regions around the target regions are displayed, with arrows showing the $5^{\prime} \rightarrow 3^{\prime}$ primer sequences.

products were not always present depending on the samples examined. Therefore, we could not determine a reliable sample genotype at this stage.

To solve this problem, we constructed two further primers; one to amplify the 3q26.1-pro-seq (60-bp fragment amplified by primer 7-8; Fig. 3 and Table III) and the other to cover the 3q26.1-pro-seq region (108-bp amplified by primer 9-10; Fig. 3 and Table III). These two primers were also used for typing together with the three previous primer pairs. In short, five kinds of PCR amplification, all producing the 141-, 71-, 294-, 60-, and 108-bp fragment, were performed for each sample to compare presence and absence of the PCR product. Again, the samples were heterogeneous with respect to the five fragments always being present, and amplification efficiency of the five fragments continued to vary among samples. We therefore arranged the method of PCR amplification as shown in Materials and methods; such that the number of PCR amplification cycles, volumes of Taq polymerase, and primer concentrations were decreased. This change in PCR conditions decreased the number of faint bands after amplification, and increased the reproducibility of amplification efficiency from the same samples. Intensity of the positive amplification products became similar among positive samples, and delicate faint bands decreased considerably in negative-like samples. This PCR amplification protocol was subsequently used to type new random samples.

We then typed 60 healthy volunteers and 54 OSCC patients. Final typing for positive (+) or negative (-) was determined by the presence or absence, respectively, of a distinct band. When a faint band was observed, PCR amplification
Table III. Oligonucleotide sequences used for PCR.

\begin{tabular}{clc}
$\begin{array}{c}\text { Primer } \\
\text { name }\end{array}$ & Type & Oligonucleotide sequence \\
\hline 1 & Forward & 5'-CGGGGAGTTTGATTTTCACT-3' \\
2 & Reverse & 5'-CGCTGTATGGTTGTCTTGTTG-3' \\
3 & Forward & 5'-GTAACACACAGCTCTGATAT-3' \\
4 & Reverse & 5'-GCCACTATAAATGCCATGAT-3' \\
5 & Forward & 5'-TCACATCAGAATTATCTGACC-3' \\
6 & Reverse & 5'-CACGTCTATGGAATTAACA-3' \\
7 & Forward & 5'-AGCGTTCTCATAATCATGGC-3' \\
8 & Reverse & 5'-AAACCGAAGTTAAGGAAAGT-3' \\
9 & Forward & 5'-GTTCAACAAGACAACCATAC-3' \\
10 & Reverse & 5'-ATGGAATTAACAGCTGTATC-3'
\end{tabular}

was performed two more times to determine if the same result could be obtained. When a faint band was reproduced three times, we considered the sample as positive $(+)$, but marked that the band intensity was weak. When the faint band was not reproducible, it was described as such. The results of typing are shown in Fig. 4 and Table IV. Identical results were obtained with the five different PCR products in most subjects $(89 \%$ of 114 subjects). However, 6 samples from each group (healthy individuals and OSCC patients) showed variation among the different PCR products. They were $(+)$ for a 71-bp product (produced by primer 1-2) and the 141-bp product (produced 


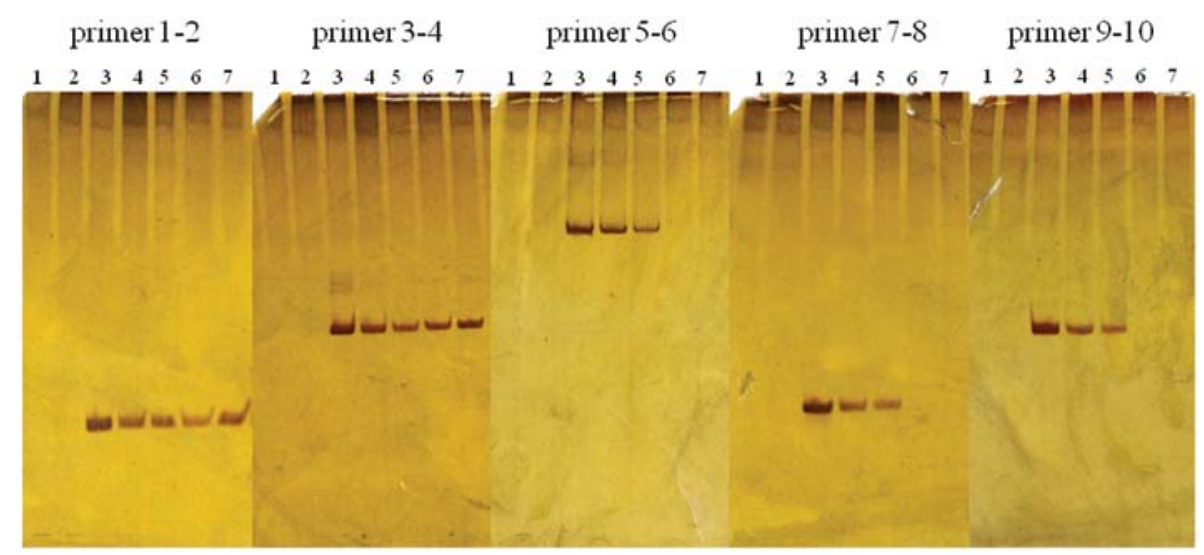

Figure 4. DNA polymorphism analysis. Native-gel electrophoresis after silver staining. Channels 1-7 show electrophoresis from different individuals. DNA samples from seven individuals were amplified using primer pairs 1-2,3-4, 5-6, 7-8, and 9-10. Channel 1 and 2 are negative for all primer pairs. Channels 3, 4, and 5 are positive for all primer pairs. Individuals 6 and 7 are positive for primer pairs 1-2 and 3-4, but negative for primer pairs 5-6, 7-8, and 9-10.

Table IV. Results of different sized PCR products.

\begin{tabular}{lcccccc}
\hline Amplified product size (bp) & 71 & 141 & 294 & 60 & 108 & \\
Combination of primer no. & $1-2$ & $3-4$ & $5-6$ & $7-8$ & $9-10$ & No. of samples \\
\hline Healthy subject & $(+)$ & $(+)$ & $(+)$ & $(+)$ & $(+)$ & 30 \\
& $(+)$ & $(+)$ & $(-)$ & $(-)$ & $(-)$ & 6 \\
OSCC subject & $(-)$ & $(-)$ & $(-)$ & $(-)$ & $(-)$ & 24 \\
& $(+)$ & $(+)$ & $(+)$ & $(+)$ & $(+)$ & 13 \\
& $(+)$ & $(+)$ & $(-)$ & $(-)$ & $(-)$ & $6^{\mathrm{a}}$ \\
\hline
\end{tabular}

${ }^{a}$ One of these samples were (-) for amplification by primer pair 1-8.

by primer 3-4), but (-) for the 294-, 60-, and 108-bp products (produced by $5-6,7-8$, and $9-10$, respectively) (Table II). Among these samples, 2 out of 6 (-) samples in the OSCC patient group showed amplification of 71- and 108-bp products as faint bands in the three PCR trials. Finally, the 12 variable samples were further amplified using three primer pairs, 1-4, $1-8$, or 1-10. All but one of the samples was (+) for the products amplified by primer pair $1-4$, but negative for those by primer pairs 1-8 and 1-10. The exception was obtained from an OSCC patient, who was (-) for all primer pairs (Table IV).

Comparison of gene frequencies between healthy volunteers and OSCC patients. As mentioned above, typing of each individual was different depending on the primers used. Because this variability could be due to genetic polymorphism, gene frequency was estimated in the healthy individuals and OSCC patients. Different genes were hypothesized depending on the size of fragments. The gene frequencies were estimated by the presence and absence of the 71-bp fragment produced by primer pair 1-2 and the 108-bp fragment produced by primer pair 9-10. Because we could not discriminate homozygote and heterozygote by the present method, we regarded (+) individuals as a dominant type composed of homozygote $(+) /(+)$ and heterozygote (+)/(-) types, and (-) individuals as a recessive genotype composed of negative homozygotes $(-) /(-)$.
Gene frequencies for the 71-bp fragment were $0.368 \pm 0.050$ and $0.632 \pm 0.050$ for the 71-bp (+) and (-) genes, respectively, in the healthy population, with $0.195 \pm 0.052$ and $0.805 \pm 0.052$ calculated as the frequencies of the 71-bp (+) and (-) genes, respectively, in the OSCC patient population. This difference in gene frequencies between healthy and OSCC patient populations was significant $\left(\chi^{2}=8.449, \mathrm{df}=1,0.01<\mathrm{P}<0.001\right)$.

Gene frequencies for the 108-bp fragment were $0.293 \pm 0.046$ and $0.707 \pm 0.046$ for the 108-bp (+) and (-) genes, respectively, in the healthy population, with $0.129 \pm 0.033$ and $0.871 \pm 0.033$ for the 108-bp (+) and (-) genes, respectively, in the OSCC patient population; also a significant difference $\left(\chi^{2}=9.209, \mathrm{df}=1\right.$, $0.01<\mathrm{P}<0.001)$. These results suggested that genetic variation of a region at chromosome $3 \mathrm{q} 26.1$ is associated with OSCC, and absence of this region was more common in OSCC patients.

\section{Discussion}

In the present study, we used a simple method for PCR amplification and detection by native gel electrophoresis to detect genetic variation of the polymorphic region found previously on chromosome 3q26.1 (3q26.1-pro-seq) in OSCC samples. To confirm the presence or absence of the region by PCR, we designed primers to amplify larger fragments containing the region identified by real-time PCR. We expected apparent variation in the presence 
or absence of amplified products irrespective of the difference in the positive homozygote or hemizygote pattern. However, amplification was not stable even between replicates of the same samples. We first suspected contamination occurring during the PCR amplification or genomic DNA isolation steps, and took steps to eliminate this possibility. We changed all reagents used for PCR amplification, conducted dual PCR of the blind PCR products, and synthesized new primers at a different company. However, contamination was suspected only in the process of dual PCR amplification of control water solution in some experiments. In these cases, contamination was not detected in the first PCR amplification. We then isolated DNA from selected subjects by the classical method of ethanol precipitation to test for contamination with foreign DNA, but comparison of PCR amplification profiles for the 141-bp fragment showed no difference between samples isolated by the two procedures. We concluded that contamination was not responsible for the variable amplification of faint bands in the present study.

We therefore decided to control the method of PCR amplification to avoid excessive amplification of the PCR products. Although approximately $90 \%$ of the samples showed clear-cut results concerning presence or absence of the five different-sized PCR products among samples from the same subjects, approximately $10 \%$ of the subjects showed different PCR amplification profiles depending on the primer pairs used for amplification. Faint and unstable PCR products are amplified when highly degraded or small amounts of DNA are used as templates for PCR amplification in common forensic cases or when primer sequences do not match sufficiently. We therefore concluded that in some of the deletion genotypes, breakage points might exist near the 3q26.1-pro-seq, with the most possible region covered by primer 4-10. In addition, breakage points may not always be identical among individuals, because amplification efficiencies differ among primer pairs. Based on these observations, we finally selected two regions (71-bp fragment amplified by primer pair 1-2 and 108-bp fragment amplified by primer pair 9-10) to determine presence or absence of the defect region in this study.

Significant differences were observed in the frequencies of polymorphism of the region around the 3q26.1-pro-seq between the healthy volunteers and OSCC patients, suggesting that a deletion polymorphism in this region (chromosome 3q26.1) could be associated with occurrence of OSCC. We also tested the association between presence and absence of the amplified products and clinical characteristics of OSCC patients. However, no statistically significant association was observed.

The 3q26.1 region was previously reported to harbor colorectal cancer susceptibility loci in earlier studies, but no candidate gene was identified. This region contains an element that regulates the expression of an upstream candidate tumor suppressor, PPM1L, thus providing a novel mechanism for colorectal tumorigenesis in APC mutation-negative familial colorectal cancer (20). Searches for a possible gene around the present deleted region using genome browser did not hit any candidates, and thus because there are reported defects around chromosome 3q26.1 in cancer patients, we cannot validate this association with OSCC in the present study due to insufficient patient numbers. Further studies involving large-scale sample collection and analysis are necessary to determine the reliability of our suggested association. The range of the deleted region should also be defined further to identify potential genes known to affect the development of OSCC.

\section{Acknowledgements}

This study was supported by the grant from Training program for Oncology Professionals in 9 Universities.

\section{References}

1. Ming Y, Qin X, Ping Z, et al: Correlation of NF-kappaB signal pathway with tumor metastasis of human head and neck squamous cell carcinoma. BMC Cancer 10: 437, 2010.

2. Zygogianni AG, Kyrgias G, Karakitsos P, et al: Oral squamous cell cancer: early detection and the role of alcohol and smoking. Head Neck Oncol 3: 2, 2011.

3. Krolls SO and Hoffman S: Squamous cell carcinoma of oral soft tissues: a statistic analysis of 14, 253 cases by age, sex, and race of patients. J Am Dent Assoc 92: 571-574, 1976.

4. Blot WJ and McLaughlin JK: Smoking and drinking in relation to oral and pharyngeal cancer. Cancer Res 48: 3282-3287, 1988.

5. Gupta PC, Mehta FS, Daftary DK, et al: Incidence rates of oral cancer and natural history of oral precancerous lesions in 10-year follow-up study of Indian villagers. Community Dent Oral Epidemiol 8: 283-333, 1980.

6. Petti S and Scully C: Oral cancer: the association between nationbased alcohol-drinking profiles and oral cancer mortality. Oral Oncol 41: 828-834, 2005.

7. Sankaranarayanan R and Ramadas K: Effect of screening on oral cancer mortality in Kerala, India; a cluster-randomized controlled trial. Lancet 365: 1927-1933, 2005.

8. Khanna JN and Andre NN: Oral sub mucosal fibrosis: a new concept in management. Report of 100 cases. Int J Oral Maxillofac Surg 24: 433-439, 1995.

9. Tanaka $\mathrm{S}$ and Sobue T: Comparison of oral and pharyngeal cancer mortality in five countries: France, Italy, Japan, UK and USA from the WHO mortality database (1960-2000). Jpn J Clin Oncol 35: 488-491, 2005.

10. Tezal M, Sullivan MA, Hyland A, et al: Chronic periodontitis and the incidence of head and neck squamous cell carcinoma. Cancer Epidemiol Biomarkers Prev 18: 2406-2412, 2009.

11. Toshinori O, Mikiko S and Sadahiko I: Characterization of CIAS1-VNTR polymorphism associated with essential hypertension. J Jpn Soc DNA Polymorph Res 16: 179-182, 2008.

12. Scully C, Field JK and Tanzawa H: Genetic aberrations in oral or head and neck squamous cell carcinoma (SCCHN): 1. Carcinogen metabolism, DNA repair and cell cycle control. Oral Oncol 36: 256-263, 2000

13. Sugahara K, Michikawa Y, Ishikawa K, et al: Combination effects of distinct cores in 11q13 amplification region on cervical lymph node metastasis of oral squamous cell carcinoma. Int J Oncol 39: 761-769, 2011

14. Chiaki Y, Kana T, Tsunenori T, et al: Examination of PCR protocol and utility of various body parts as a sample for sex identification in captive and wild birds. Jpn J Zoo Wildl Med 11: 43-48, 2006.

15. Trolet J, Hupé $P$, Huon I, et al: Genomic profiling and identification of high-risk uveal melanoma by array CGH analysis of primary tumors and liver metastases. Invest Ophthalmol Vis Sci 50: 2572-2580, 2009.

16. Weksberg R, Stachon AC, Squire JA, et al: Molecular characterization of deletion breakpoints in adults with 22q11 deletion syndrome. Hum Genet 120: 837-845, 2007.

17. Takahiro G, Hajime H, Tomoko I, et al: Prediction of MYCN amplification in neuroblastoma using serum DNA and real-time quantitative polymerase chain reaction. J Clin Oncol 23: 5205-5210, 2005.

18. Shinji U and Masahiro W: Activation of K-ras gene and allelic deletions on chromosome $17 \mathrm{p}$ and $18 \mathrm{q}$ carcinoma of the colon and rectum. Med J Kinki Univ 17: 237-248, 1992.

19. Samaneh B, Hamid R, Kamran G, et al: Soluble Fas might serve as a diagnostic tool for gastric adenocarcinoma. BMC Cancer 275: $10,2010$.

20. Thean LF, Loi C, Ho KS, et al: Genome-wide scan identifies a copy number variable region at 3q26 that regulates PPM1L in APC mutation-negative familial colorectal cancer patients. Genes Chromosomes Cancer 49: 99-106, 2010. 